\title{
Robotic System for Transapical Aortic Valve Replacement with MRI Guidance
}

\author{
Ming Li, Dumitru Mazilu, and Keith A. Horvath \\ Cardiothoracic Surgery Research Program, \\ National Heart, Lung and Blood Institute, \\ National Institutes of Health, Bethesda, Maryland, USA \\ \{lim2, mazilud, horvathka\} @mail.nih.gov
}

\begin{abstract}
This paper reports our work on developing a robotic surgical system for transapical beating heart aortic valve replacement (AVR) under interactive real-time magnetic resonance imaging (rtMRI) guidance. Our system integrates a real-time MRI system, a compound MRI robot, as well as an interface for the surgeon to plan the procedure and manipulate the robot. The compound robot consists of a positioning module and a valve delivery module. A 5-DOF Innomotion positioning arm provides and maintains direct access to the native aortic valve. A newly developed 3-DOF robotic valve delivery module allows the surgeon to remotely control bioprosthetic valve delivery with MRI guidance. Preliminary evaluation of the parameters of the robotic system demonstrates it can provide sufficient capability to successfully assist the surgeon.
\end{abstract}

\section{Introduction}

Transapical aortic valve replacement is a recently developed minimally invasive approach that allows the placement a bioprosthetic valve via a trocar that is inserted into the apex of the beating heart. Real-time magnetic resonance imaging (rtMRI) is considered as an ideal imaging modality for this procedure, due to its ability to continuously evaluate the delivery of the prosthesis, provide excellent views of valvular and annular anatomy, and can be performed on the beating heart without requiring ventricular unloading. Currently MRI-guided transapical aortic valve placement is a challenging cardiac procedure because of the limited space inside the MRI scanner and the distance from the surgeon to the target area which make it difficult to manipulate the instrumentation. Our goal is to develop a robotic system to assist performing this minimally invasive beating heart procedure under real-time MRI guidance.

Robotic system, such as the da Vinci system, has been successfully used in cardiac surgery in an attempt to minimize the morbidity of the surgical procedures. However the present $d a$ Vinci system is not MRI compatible. Previous work on MRI compatible robotic systems has primarily focused on image-guided percutaneous biopsies, drug injection or radiotherapy seed implantation. Various MRI compatible robotic 
systems have been researched and developed for prostate biopsy and brachytherapy [1-6], breast biopsy [7], interventional spinal procedures [8, 9], and neurosurgery [10]. This paper presents the design and development of a robot-assisted system for real-time MRI guided transapical aortic valve replacement. The system integrates imaging techniques and robotics that allows for remote implantation of a stented bioprosthetic valve.

\section{Clinical Background}

Traditional open heart aortic valve replacement requires sternotomy, cardiopulmonary bypass, and cardiac arrest, to achieve the result. To minimize the morbidity of the procedure, minimally invasive valve replacement such as percutaneous or left ventricular apical access is being investigated. Transapical approach provides a direct and short access to the native valve. Because the aortic valve lies in close proximity to both of the mitral valve and the coronary ostia, the position and orientation of the implanted valve is critical (see Fig. 1). Misalignment of the prosthesis could result in mitral valve damage or cardiac ischemia. Use of rtMRI for guidance allows physicians to monitor the progress of the procedure and also provides the ability to assess results immediately after intervention.

There are several obstacles to the current procedure. Access to the operative field inside the magnet is difficult for the surgeon. During the procedure, a surgical assistant holds a long delivery device in an appropriate position and angle in order to provide the surgeon a direct working trajectory to the aortic valve. Meanwhile the surgeon must advance the delivery device, manipulate the catheter that through the delivery device, and must inflate the balloon to deploy the valve under MRI guid- Fig. 1. Placing a bioprosthetic aortic valve via a ance. In order to manipulate the direct left ventricular (LV) apical access. Approdelivery device properly, a coordi- priate positioning of the bioprosthesis should be nated effort between the surgeon close to the native aortic annulus (left). Proper and the assistant is critical in the orientation of the bioprosthesis includes avoiding noisy MRI environment with the obstruction of the ostia of the left main coronary heart and lungs moving. In a beating (right).

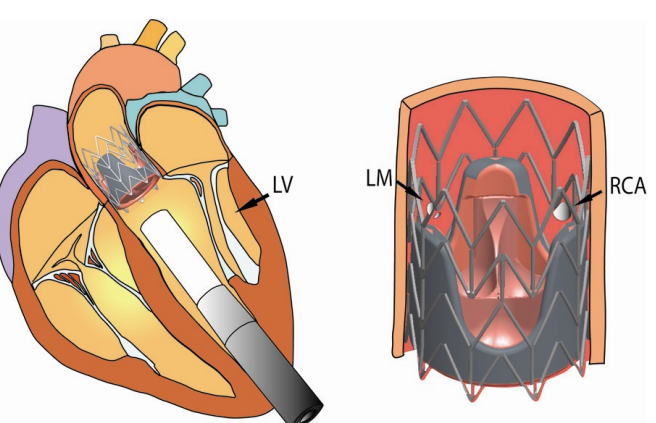
heart, the motion of the aortic annulus and the apex is typically along the long axis of the heart. As a result robotic assistance without a sophisticated tracking mechanism is applicable to aortic valve replacement via transapical approach. 


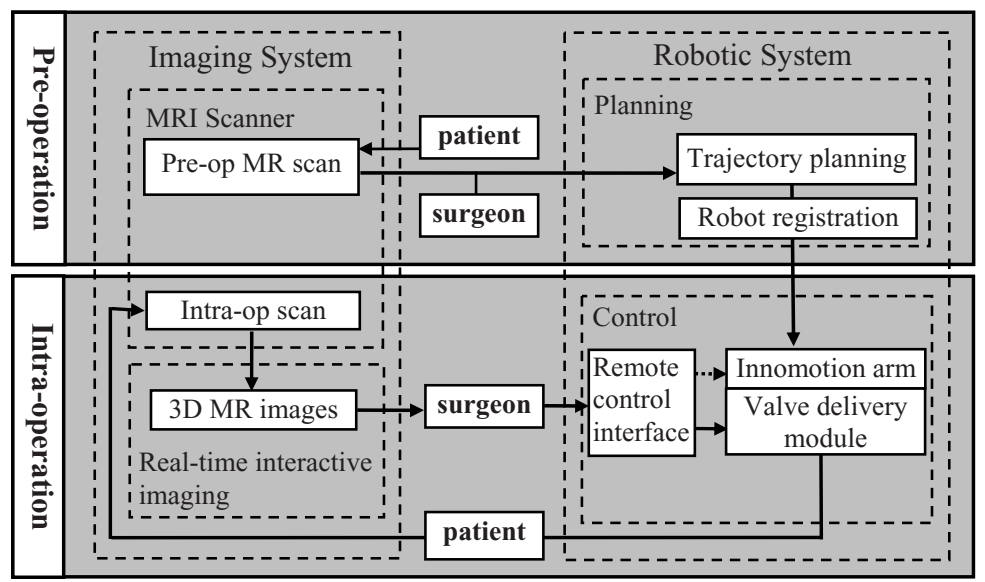

Fig. 2. System Architecture

\section{Material and Methods}

\subsection{System Architecture}

An image-guided robotic system has been designed to assist the procedure of transapical aortic valve replacement. Fig. 2 illustrates the system architecture. It comprises two major sub-systems: the imaging system and the robotic system. The imaging system consists of a standard commercial MRI scanner connected with a customized computer for real-time 3D image reconstruction. The robotic system includes a planning interface and a compound manipulator with its control interface. Preoperatively, the subject undergoes a MRI scan to determine the aortic annular diameter, coronary ostial anatomy, and apical location. The slices are sent to the planning interface of the robotic system for the surgeon to review and determine the positioning and the trajectory of the delivery device.

After calibration and registration, information from pre-operative planning is transformed into the robot coordinate frame. The positioning module is moved to the position such that the delivery device is aligned with the planned trajectory. The surgeon then controls the valve delivery module that rigidly affixed to the positioning module to manipulate the delivery device to place the valve into aortic annulus inside MRI scanner by remote control. Interactive rtMRI provides the surgeon real-time feedback of the progress.

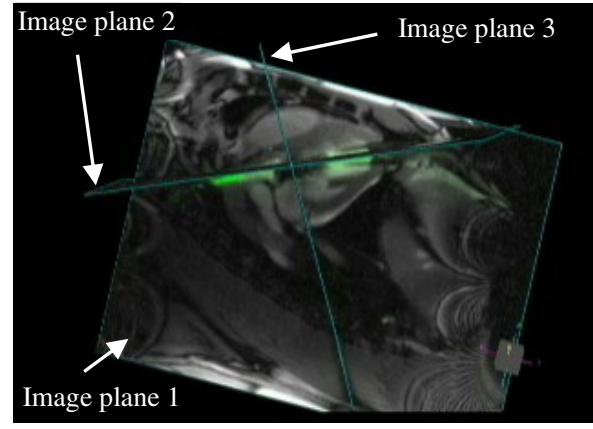

Fig. 3. Real-time multiple slice imaging with 3-D rendering on the custom reconstruction computer 


\subsection{Imaging System}

A real-time MRI interactive system [11] is used for intervention. This system consists of an interactive user interface, and operating room large screen display, specialized pulse sequences, and customized image reconstruction software. With this system, multiple (up to 3) oblique planes can be imaged and displayed simultaneously at their respective 3D locations. Three oblique image planes that used in our intervention are 1) an image plane that passes through the apex, the aortic valve and the left main coronary artery (LM), 2) an image plane that passes through the apex, the aortic valve and the right coronary artery (RCA), 3) an image plane that is on the aortic annulus. The rendering can be rotated on the in-room display to match the orientation of patient. Fig. 3 shows the 3D rendering with three image planes viewing. Image slices can be repositioned and added or omitted as needed. The MRI tissue contrast can be interactively channeled by toggling saturation pulses off/on to highlight selected objects.

\subsection{Robotic System}

Fig. 4 shows the configuration of our robotic system. It comprises two components: a 5-DOF positioning module and a 3-DOF valve delivery module. An MRI compatible Innomotion robotic arm (Innomedic, Herxheim, Germany) is employed to move and maintain the valve delivery device along with its planed trajectory. This robotic arm was originally developed for precise needle insertion for MR-guided therapy of spinal diseases [5, 9]. It has 5-DOFs with a remote center of motion (RCM) structure and its configuration fits into a standard closed MRI scanner. This fully MRI compatible robot is built of nonmagnetic materials. It is actuated by pneumatic motors and uses MR-compatible optical encoders for positioning control. In the current stage, the robotic arm is just used as a positioning tool. It can be upgraded to track the patient motion during the procedure. A newly developed 3-DOF valve delivery module is attached to the robotic arm. This compact robotic module is designed for manipulating and placing the valve into a beating heart inside MRI scanner by remote control.

\subsection{Valve Delivery Module}

We have developed a 3-DOF valve delivery module to provide precise and reproducible positioning of bioprosthetic aortic valve via transapical approach [12]. This independent module can be attached to a robotic arm or to a passive arm. The valve delivery module consists of a sterilizable valve delivery unit and an active mechanism that provides the essential manipulation of the delivery device for a valve placement. The valve delivery unit includes a delivery device, a trocar, and a trocar adaptor. The delivery device consists of a straight plastic rod, outside of which is a sheath protecting the prosthetic valve before it is deployed. The translation and rotation of the delivery device directly relate to the translation and rotation of the bioprosthesis. The active mechanism is affixed on the Innomotion robotic arm. The delivery device and 
the trocar are mounted on the active mechanism of the valve delivery module and the end-effector of the Innomotion arm respectively.

The active mechanism includes three axes: the translation axis $\underline{\mathrm{A}}$, the rotation axis $\underline{B}$ and the insertion axis $\underline{\mathrm{C}}$. The operations of these axes are independent. The order of operation is interchangeable. The translation axis provides linear displacement of the delivery device along its axis. The translation axis can travel at speeds up to $10 \mathrm{~mm} / \mathrm{sec}$ and provide $90 \mathrm{~mm}$ maximum travel length. The rotation axis allows the delivery device rotating around its axis to change the orientation of the prosthesis relative to coronary ostia before it is deployed. This axis travels at speeds up to $5 \mathrm{deg} / \mathrm{sec}$ and the travel range is \pm 60 degree. The third one is the insertion axis. It actuates the relative movement of the inner rod and sheath of the delivery device to advance the prosthesis out-

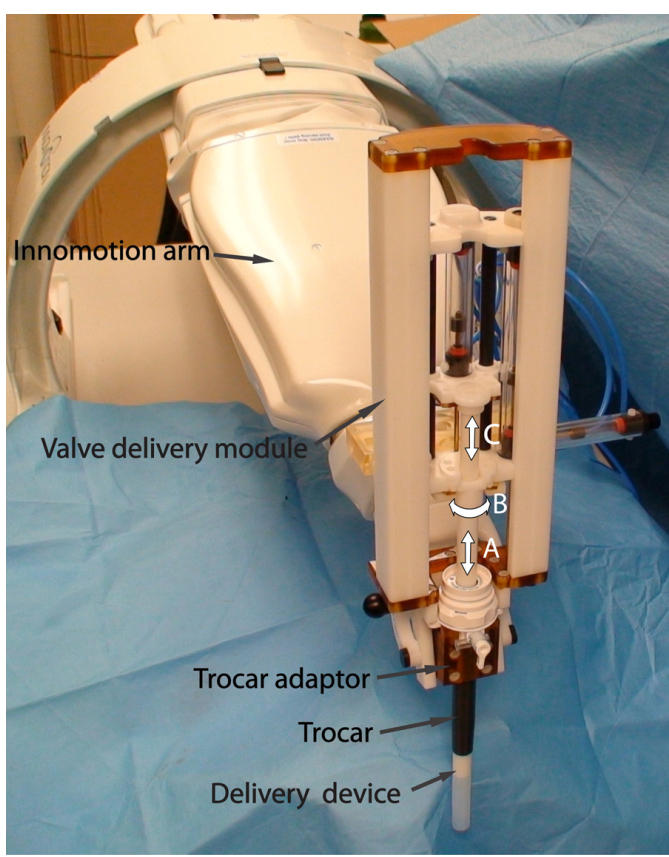

Fig. 4. Picture of the robotic system for MRI guided transapical AVR. It shows an Innomotion arm for the positioning module and the prototype of the new developed valve delivery module affixed on the robotic arm. side of the sheath.

To maintain image quality and prevent local heating in the proximity of the patient, all parts of the prototype module were made from non-conductive plastic materials such as Delrin, Ultem, and Polyetheretherketones (PEEK). Pneumatic actuators were used to actuate all three axes. The MR compatible pneumatic cylinders were made with glass bores, graphite pistons, brass shafts and plastic housings (Airpel, Norwalk, CT). Magneto-translucent fiber-optical linear and rotary incremental encoders made of glass and ceramics (Innomedic, Herxheim, Germany) were used to gather positioning information.

A 2-GHz Pentium IV IBM PC-compatible machine running Windows XP with an XMP-SynqNet-PCI hardware interface (Motion Engineering Inc, USA) installed was used for control. A PIV (proportional position loop integral and proportional velocity) controller run on the board was used for servoing the valve delivery module movement. Remote Motion Block (RMB) was used to connect conventional analog drives of the pneumatic servo valves into a SynqNet motion network system. The control PC was placed outside the MR room, and the RMB with pneumatic servo valves and other pneumatic supplies was placed outside the 5 Gauss line inside the MR room. 


\subsection{System Integration and User Interfaces}

The Innomotion robotic arm is mounted on the side-rails of the MRI table. The robotic arm is calibrated based on four fiducial markers rigidly attached to its endeffector. These fiducial markers are composed of diluted gadolinium-DTPA.

We developed a graphic user interface for the pre-operative surgical plan. The surgeon first inserts a trocar with an adaptor into the apex of the heart. With a MRI scan, MR images are sent via DICOM protocol to the control PC and planning of the device trajectory is performed by clicking on the images in the user interface. The trajectory is transformed to the registered robot coordinate frame. The surgeon loads the delivery device with the prosthetic valve and inserts the delivery device into the trocar. The robotic arm with the active mechanism of the valve delivery module affixed is moved to the position. The trocar and the delivery device are then mounted on the endeffector and the active mechanism of the delivery module respectively. Thus, direct access to the aortic valve is created. We also developed a simple interface for module control. It provides coarse and fine two-level control on the translation and rotation axes. The surgeon determines and inputs the translation and rotation values and clicks the coarse control buttons to move the prosthetic valve to the correct place, he/she can tune the position and orientation by clicking the fine control buttons. Once the bioprosthetic valve has reached the desired position, the surgeon clicks the insertion button to advance the valve out from the delivery device and inflates the balloon to deploy the valve.

\section{Results}

We have evaluated the parameters of the prototype of the valve delivery module. First the mechanical accuracy of the valve delivery module was evaluated. The result is shown in Fig. 5. The air pressure we used to actuate the pneumatic pistons was 75psi. The maximum measured force load of the translation axis was $34 N$; and the maximum torque of the rotation axis was $0.4 \mathrm{Nm}$. The module provided enough stiffness and rigidity. With operational velocity of $10 \mathrm{~mm} / \mathrm{s}$, the accuracy of translation axis was found to be $0.19 \pm 0.14 \mathrm{~mm}$. With operational velocity of $5 \mathrm{deg} / \mathrm{s}$, the accuracy of the rotation axis was found to be $0.46 \pm 0.27 \mathrm{deg}$.
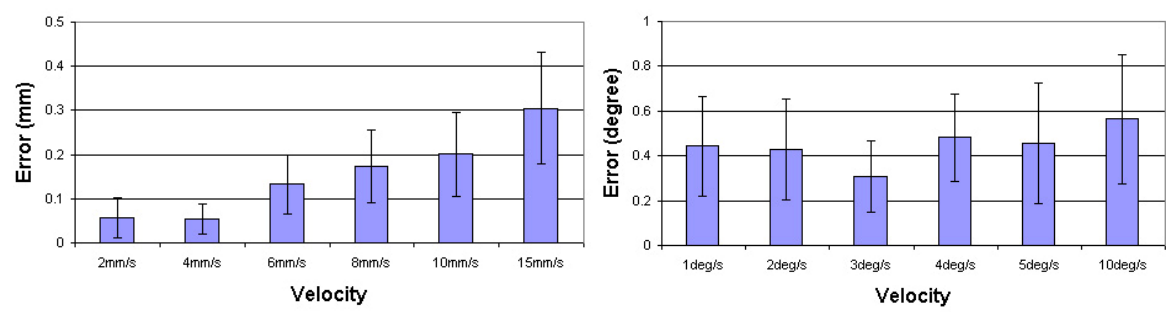

Fig. 5. Mechanical accuracy of the translation stage (left) and rotation stage (right) with different velocity. They were measured by module's encoders with $75 p s i$ air pressure supply and with the delivery unit attached. 
The MR compatibility of the valve delivery module was studied on a $1.5 \mathrm{~T}$ Siemens Espree scanner. A steady-state free precession (SSFP) sequence was used with following scanning parameter: $\mathrm{TR}=436.4 \mathrm{~ms}$, $\mathrm{TE}=1.67 \mathrm{~ms}$, echo spacing $=3.2 \mathrm{~ms}$, imaging flip angle $=45 \mathrm{deg}$, slice thickness $=4.5 \mathrm{~mm}$, field of view $(\mathrm{FOV})=340 \times 283 \mathrm{~mm}$, and matrix $=192 \times 129$. This protocol was similar to the one we used in MR scanning for the cardiac intervention. The presence and motion of the robotic device inside the scanner was found to have no noticeable disturbance in the image (see Fig. 6). The observed SNR loss was $6.1 \%$ to $6.5 \%$ for the valve delivery module placed in the scanner and the valve delivery module in motion respectively.
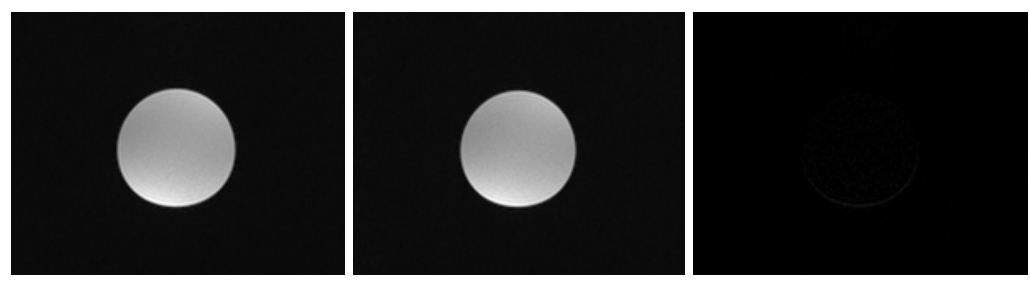

Fig. 6. Images of the cylindrical phantom when the valve delivery module was not inside MR room (left), and when the valve delivery module was in motion (middle). The delivery module was placed such that the distal end of the active mechanism was at the distance of $150 \mathrm{~mm}$ away from the center of the image area. The difference between images in (left) and (middle) is shown (right).

We also evaluated the accuracy of the Innomotion arm as an image-guided delivery device holder. A phantom was designed for this purpose. The phantom consisted of a plastic tube with $25 \mathrm{~mm}$ diameter that was mounted on one side of a $200 \times 100 \times 100 \mathrm{~mm}$ water tank served as the aorta. A spherical joint that mounted on the opposite side of the tank served as the apex. The trocar that inserted into the spherical joint can pivot on it. The phantom was scanned with the MRI. Using our planning interface, we selected the center of the tube end and the center of the spherical joint in the images to define the trajectory for the delivery device. The registered robotic arm was moved into the correct position and orientation. We mounted the trocar and delivery device on the robot and advanced the delivery device through trocar until the distal end of the delivery device reached the end of the tube under rtMRI guidance. The maximal inplane displacement error over 5 trials was $2.8 \mathrm{~mm}$. The displacement error of the delivery device was defined as the distance between the center of the distal end of the delivery device and the center of the end of the tube in the MR image. The maximal calculated angular error was $1.4 \mathrm{deg}$. The angular error was defined by the angular difference between the line that goes through the center of the end of the tube and the spherical joint, and the center line of the delivery device.

\section{Discussion and Conclusion}

We have developed a prototype robotic surgical system for transapical aortic valve replacement with real-time MRI guidance. Our system integrates interactive real-time 
MRI system, a robotic arm with a newly developed robotic valve delivery module, as well as an interface for the surgeon to plan the procedure and manipulate the robot. The maximal in-plane displacement error at the aortic valve level was found to be $2.8 \mathrm{~mm}$ using the adapted Innomotion arm in a phantom study. The mechanical accuracy of the Innomotion arm and MRI process contribute to the system level error. Within this error range, one can easily insert a prosthetic valve protected by a $12 \mathrm{~mm}$ delivery device into a typical $25 \mathrm{~mm}$ aortic annulus. Our experiments suggest that a 5DOF Innomotion positioning arm can provide direct access to the aortic valve and the newly developed valve delivery module can provide sufficient capability to successfully assist the surgeon to remotely control bioprosthetic valve delivery with MRI guidance.

Our immediate future work is to evaluate the complete system in a phantom study and in a large animal study to mimic the clinical scenario.

\section{Acknowledgment}

The authors are supported through the Intramural Research Program of the National Heart, Lung, and Blood Institute, NIH, DHHS.

\section{References}

1. Chinzei, K., et al.: MR compatible surgical assist robot: system integration and preliminary feasibility study. In: Delp, S.L., DiGoia, A.M., Jaramaz, B. (eds.) MICCAI 2000. LNCS, vol. 1935, pp. 921-930. Springer, Heidelberg (2000)

2. Fichtinger, G., et al.: Transrectal Prostate Biopsy Inside Closed MRI Scanner with Remote Actuation, under Real-Time Image Guidance. In: Dohi, T., Kikinis, R. (eds.) MICCAI 2002. LNCS, vol. 2488, pp. 91-98. Springer, Heidelberg (2002)

3. Fischer, G.S., et al.: Development of a Robotic Assistant for Needle-Based Transperineal Prostate Interventions in MRI. In: Ayache, N., Ourselin, S., Maeder, A. (eds.) MICCAI 2007, Part I. LNCS, vol. 4791, pp. 425-453. Springer, Heidelberg (2007)

4. Krieger, A., et al.: Design of a novel MRI compatible manipulator for image guided prostate interventions. IEEE Trans. Biomed Eng. 52, 306-313 (2005)

5. Munterner, M., et al.: Magnetic resonance imaging compatible robotic system for fully automated brachytherapy seed placement. Urology 68, 1313-1317 (2006)

6. Stoianovici, D., et al.: MRI Stealth robot for prostate interventions. Minim Invasive Ther Allied Technol. 16, 241-248 (2007)

7. Kaiser, W.A., et al.: Robotic system for biopsy and therapy of breast lesions in a high-field whole-body magnetic resonance tomography unit. Invest Radiol. 35, 513-519 (2000)

8. Hempel, E., et al.: An MRI-compatible surgical robot for precise radiological interventions. Computer Aided Surgery, 180-191 (2003)

9. Melzer, A., et al.: MRI and CT Compatible Robotic System for Percutaneous Image Guided Interventions: Principles and Evaluation. IIIE Engineering in Medicine and Biology (2008) 
10. Masamune, K., et al.: Development of an MRI-compatible needle insertion manipulator for stereotactic neurosurgery. J. Image Guided Surgery 1, 242-248 (1995)

11. Guttman, M., et al.: Real-time accelerated interactive mri with adaptive sense and unfold. Magn. Reson. in Med. 50, 315-321 (2003)

12. Li, M., et al.: A novel robotic valve delivery module for transapical aortic valve replacement under MR guidance. IEEE BioRob (submitted, 2008) 